\title{
L'influence De La Professionnalisation De La GRH Sur La Valorisation Du Métier De L'enseignant- Chercheur : Le Rôle De L'approche Compétence, Cas Des Enseignants Des Grandes Écoles Revue De Littérature Et Essai De Proposition D'un Cadre Conceptuel
}

\author{
Sarah Chetouani (Doctorante) \\ M. Saïd Balhadj (Directeur de Thèse)
}

Groupe de Recherche : Management et Audit des Organisations

Université Abdelmalek Essaadi, ENCG Tanger, Maroc

Doi: 10.19044/esj.2017.v13n34p186 URL:http://dx.doi.org/10.19044/esj.2017.v13n34p186

\begin{abstract}
Teaching in universities is currently the main subject of this study, whereby the notions of professionalization and academic pedagogy is considered. The desire to promote the profession of university professor is remarkable as it comes against logics of recognition that are not very compatible. For this reason, particular attention must be paid to the human resource management policy concerning university professors in all aspects of their professional life: their careers, their training needs, their working conditions, and so on. It must also be thought out and focused on results and competitiveness, particularly with regard to the attractiveness and management of positions. This is with a view to ensure better professionalization of the public it trains. The originality of this paper lies in proposing a conceptual framework of reflection and analysis. It aims to understand to what extent the valorization of the profession depends on the professionalization of the HR practices through the setting up of a true competency approach. This approach aims at defining the skills profile of the Moroccan university professors who will serve to better guide and professionalize human resources management activities. Also, the study aims to replace pedagogy as the fundamental competence of the profile of university professor at the heart of the teaching-learning processes of the Moroccan higher education schools.
\end{abstract}


Keywords: Professionalization, Human Resources Management, competency approach, skills profile, university professors, university pedagogy

\section{Résumé}

Enseigner à l'université est actuellement l'objet de questionnements dans lesquels surgit les notions de professionnalisation et de pédagogie universitaire. La volonté de valoriser le métier de l'enseignant-chercheur est d'autant plus remarquable qu'elle se heurte à des logiques de reconnaissance peu compatibles. Pour cette raison, une attention particulière doit être portée à la politique de gestion des ressources humaines concernant les enseignants universitaires dans tous les aspects de leur vie professionnelle: leurs carrières, leurs besoins en formation, leurs conditions de travail, etc. Elle doit également être pensée et orientée résultat et coméptence notamment pour ce qui concerne l'attractivité et la gestion des postes en vue d'une meilleure profesionnalisation des publics qu'elle forme. L'originalité de ce papier réside dans le fait de proposer un cadre conceptuel de réflexion et d'analyse visant à comprendre dans quelle mesure la valorisation du métier de l'enseignant-chercheur dépend de la professionnalisation des pratiques RH à travers la mise en place d'une véritable approche compétence visant la définition du profil de compétences de l'enseignant-chercheur marocain qui servira à mieux guider et professionnaliser les activités de gestion des ressources humaines. Aussi, il voudrait replacer la pédagogie-comme étant la compétence fondamentale du profil d'un enseignant-chercheur- au cœur des processus d'enseignement-apprentissage des grandes écoles d'enseignement supérieur marocaines.

Mots-clés : Professionnalisation, GRH, approche compétence, profil de compétences, enseignants-chercheurs, pédagogie universitaire

\section{Introduction}

Le monde de l'enseignement supérieur est aujourd'hui marqué par la nécessité de performance et d'efficience en matière de recherche et d'enseignement. En effet, le dynamisme et la cohérence d'action des établissements d'enseignement supérieur et de recherche reposent sur les compétences et la mobilisation de leurs personnels. Parmi ceux-ci, les femmes et les hommes qui composent l'université et qui en constituent le potentiel, la force et la richesse. Permettre à ce potentiel de s'exprimer pleinement exige de l'attention, des moyens, des structures, en d'autres termes, d'une politique personnalisée de la ressource enseignante spécifiquement orientée compétence et résultat, qui visera donc à dépasser les simples actes de "gestion du personnel », pour rechercher la 
professionnalisation et la production d'un nouveau discours portant sur des compétences nouvelles et leur développement pour les enseignantschercheurs marocains. Cette démarche impose préalablement la clarification des choix et une réflexion prospective sur l'évolution des compétences. Elle suppose la mobilisation des outils classiques de la gestion des ressources humaines au service du projet ainsi défini. Elle ne peut atteindre son objectif qu'à la condition que les attentes des individus se retrouvent dans le projet d'ensemble.

Pour cette raison une attention particulière doit être portée à la politique de gestion des ressources humaines concernant les enseignantschercheurs de l'enseignement supérieur. Cette gestion les concerne naturellement dans tous les aspects de leur vie professionnelle : leurs carrières, leurs besoins en formation, leurs conditions de travail, etc. Elle doit également être pensée et dirigée au service de la stratégie de l'établissement, notamment pour ce qui concerne l'attractivité et la gestion des postes.

En effet, une tendance actuelle amène la fonction publique à se transformer selon la gestion des compétences. En tant que fonctionnaire, l'enseignant-chercheur universitaire pourrait être visé par cette mouvance qui marque sans ambiguïté une volonté de mieux faire contribuer les institutions concernées au développement économique et social. Enseigner à l'université est actuellement l'objet de questionnements dans lesquels surgit la notion de professionnalisation. La volonté de valoriser le métier de l'enseignantchercheur en développant une approche compétence est d'autant plus remarquable qu'elle se heurte à des logiques de reconnaissance peu compatibles : l'évaluation des enseignants est centrée principalement sur la recherche et l'investissement pédagogique est peu valorisé.

Or, il n'existe aucune description d'emploi, aucune description de tâches ni de profil de compétences précisant les indicateurs à partir desquels un enseignant de niveau universitaire devrait être évalué ou encore guidé dans le développement de ses compétences.

Sur ce registre, en tentant un bilan raisonné de l'état de l'art sur cette problématique d'importance, en faisant un diagnostic objectif et pertinent des aspects les plus saillants de la GRH des enseignants-chercheurs marocains et en ouvrant des perspectives opérationnelles pour un avenir plus prometteur, l'objectif de cette recherche est de tenter d'expliquer dans quelle mesure la valorisation du métier de l'enseignant-chercheur dépend de la professionnalisation des pratiques $\mathrm{RH}$ à travers la mise en place d'une véritable approche compétence visant la définition du profil de compétences de l'enseignant-chercheur qui servira à mieux guider et professionnaliser les activités des gestion des ressources humaines. Aussi, il voudrait replacer la pédagogie -comme étant la compétence fondamentale du profil d'un 
enseignant universitaire - au cœur des processus d'enseignementapprentissage des grandes écoles d'enseignement supérieur marocaines.

Cette recherche constituera donc un apport réel quant à la compréhension de la question de professionnalisation de la GRH, comme élément de valorisation et d'amélioration de l'attractivité du métier de l'enseignant chercheur. En d'autres termes, une approche compétence du management des ressources humaines personnalisée aux enseignants des grandes écoles va ouvrir une véritable perspective opérationnelle aux décideurs, mais aussi la méthodologie que nous allons développer, constitue une des principales contributions de cette recherche-action.

\section{Valorisation Du Métier De L'enseignant-Chercheur: Analyse De La Littérature \& Cadrage Conceptuel \\ L'enseignant-chercheur de l'université}

Dès le début de sa carrière, l'enseignant-chercheur doit rencontrer des besoins en qualificiations incontournables: former des jeunes souvent en grands groupes (conditions de travail difficiles) à propos de contenus à (re) construire pour eux. Bien souvent bâtir et rédiger des notes de plusieurs cours sont certains pour lesquels le nouveau professeur n'est pas « également » familier. D'un point de vue affectif, il ressent, du moins en début de carrière, testé etc. Bien souvent, il se sent isolé pour remplir, dès le départ, une tâche complexe.

Plus tard et de plus en plus, ses tâches ne se limiteront pas à enseigner et il sait qu'il sera « évalué » sur ses productions scientifiques et sa participation aux tâches institutionnelles et de service à la collectivité. Bien souvent, il lui sera demandé de développer un secteur de recherche si possible «nouveau et en pointe ». Il doit, c'est devenu une nécessité, participer à un ou plusieurs réseaux européens d'échange avec des universités prestigieuses. Quoi d'étonnant que ce professionnel « multitâches » ressente des «tensions » entre ces différentes fonctions et activités à mener à bien sous le regard de ses collègues et étudiants. Ces remarques ne tiennent évidemment pas encore compte des enjeux institutionnels et individuels particuliers au contexte de chaque professeur d'université.

Enfin, si le professeur d'université a choisi ce métier, c'est sans doute parce qu'il a cru pouvoir y exprimer son individualité dans un certain espace de liberté. Il devra trouver dans cette fonction multitâches le moyen d'être ce qu'il est. La recherche (individuelle ou en équipe) est très souvent perçue comme le meilleur moyen de réaliser son individualité, sans doute parce que les contraintes liées à la recherche dépendent, à ses yeux, plus de la personne du chercheur lui-même que des autres facteurs. De plus, la reconnaissance académique est basée sur ses performances en tant que chercheur. 
Pour réaliser son projet professionnel, il doit cependant tenir compte de plus en plus de contraintes liées aux concurrences intra et interinsitutionnelles : les postes définitifs se font plus rares en même temps que le nombre de candidats augmente. Le nombre d'étudiants ne cesse de croître dans les mêmes conditions matérielles et temporelles. Les évaluations institutionnelles de la «qualité » deviennent plus systématiques et sont placées dans les mains "d'experts", les modèles industriels classiques entrent dans les insitutions publiques. Nous pourrons allonger la liste des contraintes nouvelles qui ont « changé le métier » d'enseignant-chercheur.

La grande majorité des professeurs d'université marocaines ressentent assez mal le manque de temps à consacrer à ce qu'ils estiment être une de leur tâche essentielle : créer du savoir scientifique.

Parrallèlement, la pression sociale s'exerce sur l'université lui reprochant un taux d'échec invariablement ravageur, en même temps qu'une «baisse de niveau » et un manque de préparation à la vie professionnelle, sans compter que le diplôme universitaire n'offre plus la garantie de l'emploi.

J. Donnay et M. Romainville ont proposé un modèle reflétant la situation de travail de ce professionnel multitâches :

Figure 1. Modèle de situtation de travail du professeur d'université

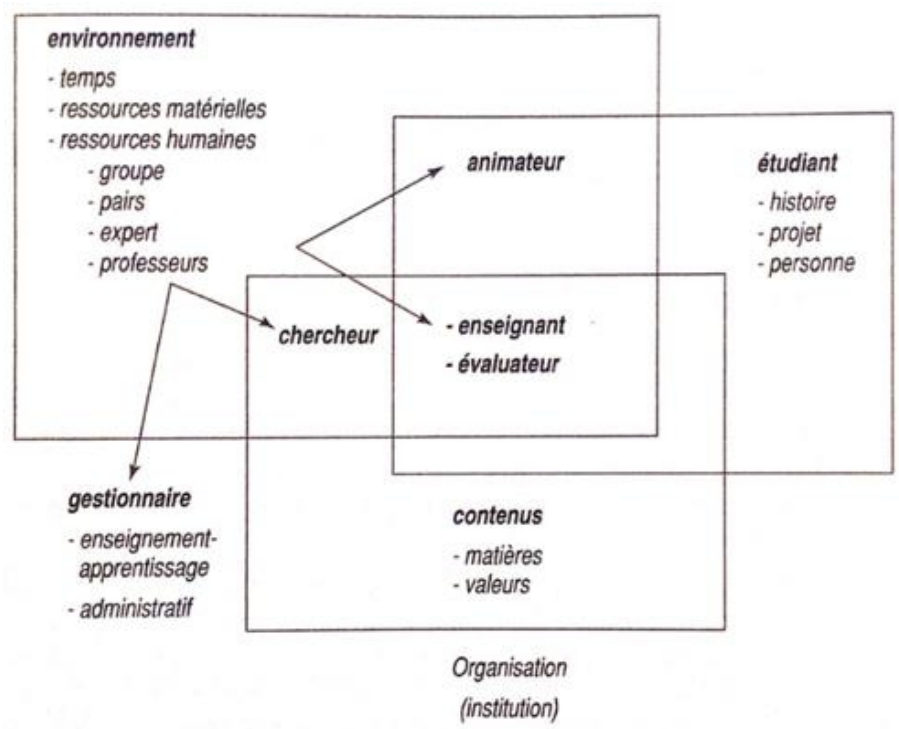

Source: J. Donnay et M. Romainville (1996)

C'est dans ce contexte qu'il est demandé à l'enseignant-chercheur " d'améliorer sa pédagogie », tâche pour laquelle il n'a été généralement ni formé, ni choisi. La pédagogie à l'université se situe au cœur de ces tensions vécues plus ou moins bien par tout enseignant-chercheur. 
Pour J. Donnay et M. Romainville (1996), la valorisation de ce métier repose principalement sur une approche pragmatique de valorisation de l'invesissement pédégogique des enseignants-chercheurs qui tient autant que possible des « conditions de travail » de ces derniers.

\section{L'amélioration de la pédagogie à l'université : Outil incontournable de valorisation du métier de l'enseignant-chercheur}

Bon nombre de professeurs enseignent selon le modèle pédagogique le plus répandu, basé sur le transfert de connaissance. Sont-ils autre choses qu'un intermédiaire : d'abord, entre des savoirs et des valeurs, d'une part, et des étudiants qui sont censés les maîtriser d'autre part, et entre ces mêmes étudiants d'un côté et des ressources disponibles, un environnement pour apprendre de l'autre (Figure 1)?

J. Donnay et M. Romainville ont tenté de schématisé quelques éléments susceptibles d'intervenir dans une politique de formation pédagogique. L'association des deux figures (2 et 3 ) combine deux identités : l'identité institutionnel de l'université et ses interactions avec l'environnement dans lequel il s'insère (Figure 2) et l'identié professionnelle de chacun des des professeurs d'université (Figure 3) reposant sur sa perception des composantes de ses fonctions, de sa personne, de l'institution et de l'environnement dans lequel il s'insère. Ces deux grands registres de « paramètres » constituant les identités, interagissent (processus) pour influencer la perception de la pédagogie universitaire (produit) que se fait le professeur d'université. La pédagogie est-elle une contrainte ? Une opportnité de recherche, de création, un outil ? Cette perception est sans doute un des éléments à prendre en compte en vue de bâtir une politique de la formation pédagogique des professeurs. En effet, par exemple, on observe intuitivement une relation entre l'image extérieure donnée par l'institution au point de vue de sa politique pédagogique de l'enseignement, ses relations avec le public cible étudiants et les pouvoirs politiques nationaux, entre le recrutement et la valorisation du personnel académique d'une part, la perception qu'a le professeur de ses fonctions et de son investissement dans sa fonction de formateur, d'autre part. Chaque axe de la perception du professeur se présente sous forme d'une tension entre deux (ou plusieurs) pôles contradictoires sous l'un ou l'autre aspect ; par exemple : tension entre une conception quantitative et/ou qualitative de l'apprentissage. C'est à chacun de gérer tant bien que mal ses tensions et d'adopter plus ou moins consciemment une attitude jugée adéquate.

Le but des deux auteurs à travers les schémas présentés ci-dessous est donc de se donner un premier outil de découpage ou d'aide à la prise de décision en termes de politique de formation pédagogique des professeurs d'université en vue de valoriser et d'améliorer l'attractivité du métier. 
Figure 2. Éléments de l'identité insitutionnelle susceptible d'intervenir sur la valorisation du métier de l'enseignant-chercheur

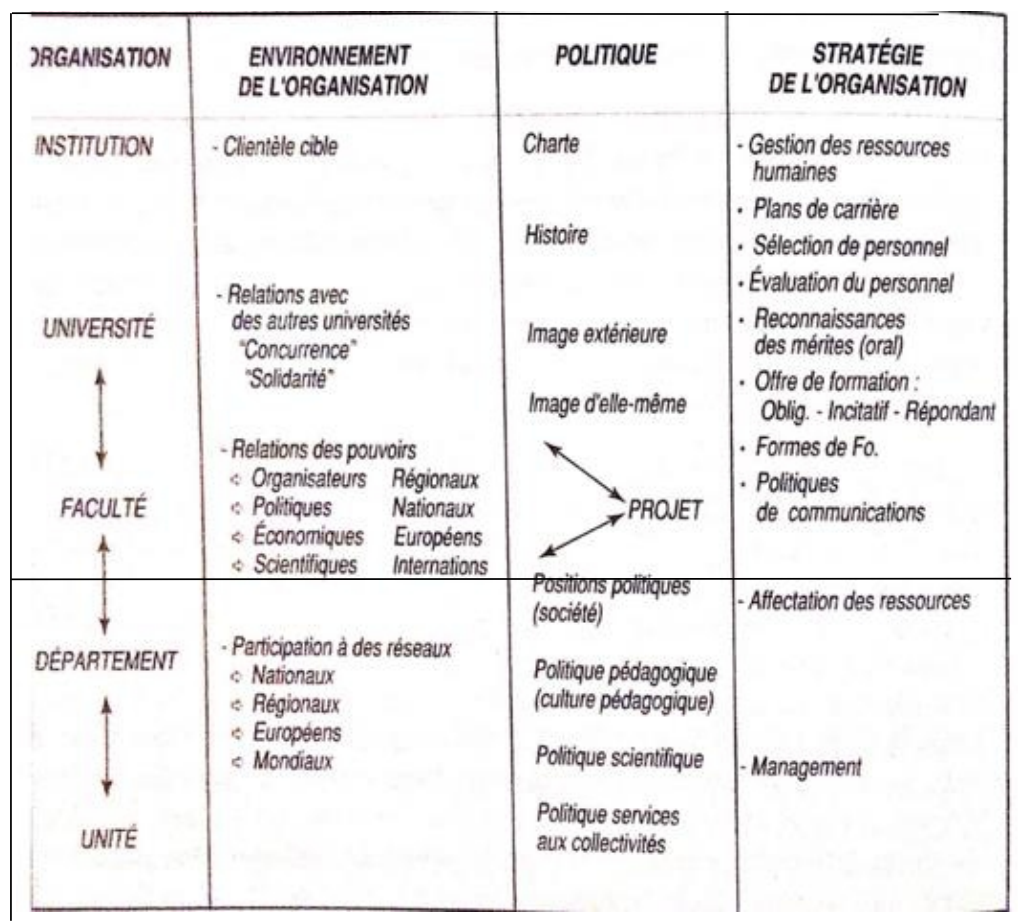

Source: J. Donnay et M. Romainville (1996)

Figure 3. Eléments de l'identité professionnelle susceptibles d'intervenir dans la valorisation du métier de l'enseignant-chercheur

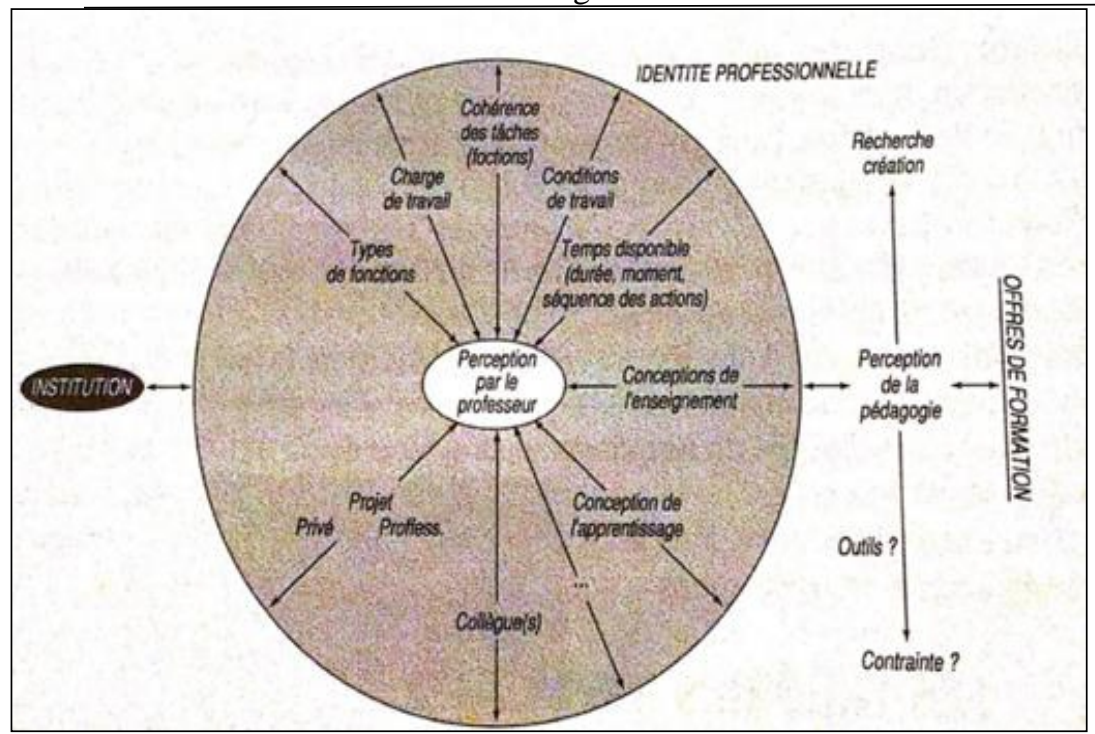

Source: J. Donnay et M. Romainville (1996) 
Plus que les facteurs en eux mêmes, c'est la perception que peut en avoir le professeur d'université qui déterminera son degré d'implication dans une politique de ressources humaines en générale et dans une politique de formation touchant à son métier de formateur plus particulièrement.

J. Donnay et M. Romainville (1996) pensent que l'identité professionnelle du professeur d'université, et en particulier sa représentation de la pédagogie, peut être considérée comme la résultante de ses perceptions d'un faisceau de facteurs (voir Figures 1, 2, 3) intervenant dans ses fonctions, s'inscrivant elles-mêmes dans l'institution et dans le temps. Ce qui signifie que ses perceptions seront contextualisées et évolutives.

La littérature dans le domaine nous a permis de retenir quelques invariantes. Il nous semble qu'une approche pédagogique reposant sur les conceptions que le professeur se fait de son rôle (fut-il changeant), de l'enseignement et de l'apprentissage et une explicitation de celles-ci est un point d'entrée privilégié. L'évocation, l'explicitation, la description, l'analyse de ces conceptions ou des situations concrètes, leur confrontation à d'autres possibles sont d'ailleurs quelques composantes d'une formation à la réflexivité. L'analyse des pratiques concrètes et leur confrontation à d'autres pratiques, même de collègues d'autres disciplines, constituent un réservoir extrêmement riche d'ouverture non seulement à d'autres possibles, mais aussi à une théorisation (explicitation de théorie personnelles pratiquées) perçue comme utile et valorisante. C'est ici que se rejoignent les attitudes de recherche du formateur et la qualité de chercheur du professeur, la formation à la réflexivité leur servant de trait d'union. Cette démarche réflexive s'inscrit en outre dans le concret puisqu'elle prépare à la décision et au passage aux actes quotidiens.

L'essentiel de la formation pédagogique des enseignants du supérieur ne devrait-il dès lors pas consister en l'explication, la confrontation et l'enrichissement de leurs concceptions des actes mêmes d'apprendre et d'enseigner? Des recherches visant à décrire en détails des conceptions commencent d'ailleurs à se multiplier. Très souvent, elles aboutissent à des typologies qui pourraient être également utilisées comme outis de formation (Ramsden, 1992; Dall Alba, 1993; Samuelowicz \& Bain, 1992; Trigwell et al., 1994; Prosser, 1994).

\section{Le SoTL, un modèle théorique de développement professionnel et d'amélioration de l'attractivité du métier de l'enseignant-chercheur}

Dans les années 90, Boyer modélise la fonction de professeur d'université : activités de recherche, publications, services d'expertise rendus à la société ainsi qu'expertise en enseignement ou Scholarship of Teaching (Rege Colet, McAlpine, Fanghanel \& Weston, 2011; Thériault, 2011). Bien vite, le concept évolue. Le focus n'est plus seulement centré sur 
l'enseignement, mais également sur l'apprentissage : Scholarship of Teaching and Learning (SoTL).

Soutenus par le développement d'Internet, des moyens de communication et de "revues savantes libres », nombre de chercheurs de différentes disciplines collaborent et alimentent la réflexion sur les pratiques pédagogiques dans l'enseignement supérieur (Thériault, 2011). Toute une communauté dispersée, tant sur le plan géographique que sur le plan disciplinaire, contribue ainsi à l'essor du SoTL. Basé sur l'idée que l'expertise en enseignement-apprentissage permet autant d'améliorer l'apprentissage des étudiants que l'enseignement (d'abord de l'expert concerné, ensuite de toute la communauté enseignante par répercussion), le SoTL constitue à la fois un modèle de développement professionnel et un outil d'amélioration de la qualité de l'enseignement supérieur.

Plusieurs auteurs (Ashwin \& Trigwell, 2004; Kreber \& Cranton, 2000; McKinney, 2007; Smith, 2001; Weston \& McAlpine, 2001) se sont intéressés à cerner les stades du processus de transformation continue de la pratique enseignante vers le SoTL et ont proposé des modèles développementaux pour rendre compte des manifestations particulières à chacun des stades :

Bélanger (2010) présente les trois principales étapes pour atteindre cette expertise en enseignement-apprentissage. Il s'agit, d'abord, d'une réflexion sur l'enseignement et l'apprentissage, une articulation aux connaissances pédagogiques, nourrie de discussions et d'échanges avec les pairs. Ensuite, cette réflexion s'appuie sur des investigations : la pratique et son analyse sont documentées, notamment pour cerner les impacts de l'enseignement sur l'apprentissage. Enfin, la diffusion des résultats dans des colloques ou revues favorise une répercussion des découvertes sur l'ensemble de la communauté des enseignants du supérieur.

Ces trois étapes sont reprises dans la Figure 5 ci-dessous. Le SoTL implique une démarche systématique d'analyse et de modification de la pratique enseignante dans laquelle l'importance accordée à l'action et à la transformation est cruciale (Rege Colet et al., 2011). 
Figure 4. Le SoTL: les phases et les processus

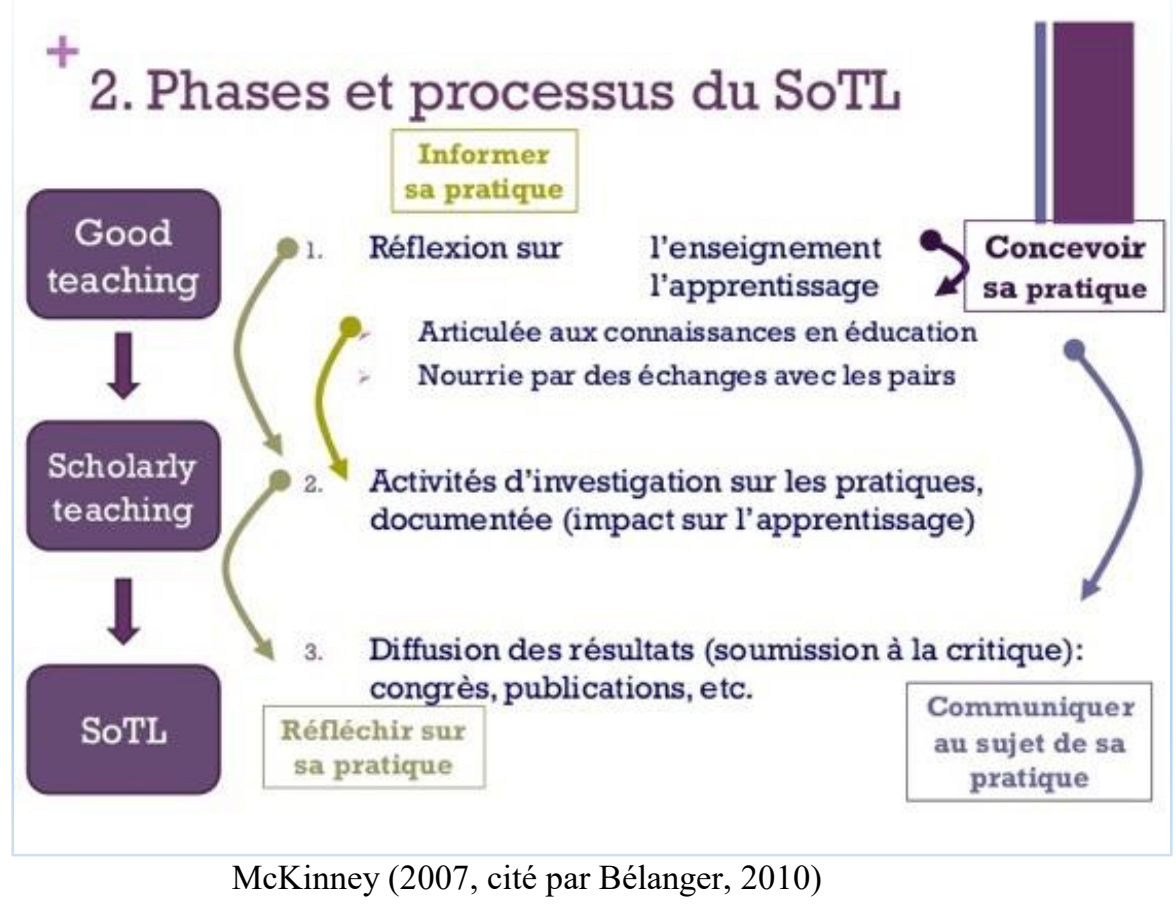

Ce schéma tente d'articuler les phases et les processus du développement professionnel. Il s'inspire notamment des trois phases établies par McKinney (2007, cité par Bélanger, 2010, p. 4) et caractérisées par Vajoczki, Savage, Martin, Borin et Kustra (2011):

- Good teaching ou l'enseignement efficace selon sept indicateurs:

1) contacts entre la faculté/l'institution et les étudiants ;2) coopération entre étudiants ; 3) utilisation de pédagogies actives ; 4) feedback rapide ; 5) temps passé sur la tâche; 6) attentes élevées ; 7) utilisation de différentes voies d'apprentissage ;

- $\quad$ Scholarly teaching ou l'enseignement rigoureux et réflexif: ce type d'enseignement implique la consultation de la littérature, la sélection d'éléments pertinents pour la pratique, des observations systématiques, une analyse de résultats ou une évaluation par les pairs ;

- $\quad$ SoTL ou expertise en enseignement-apprentissage: caractérisé par un effort pour comprendre comment les étudiants apprennent effectivement et de quelle manière l'enseignement influence cet apprentissage. Le SoTL implique un apprentissage au sujet de son enseignement et un partage public des résultats de cet apprentissage.

Aux trois phases du SoTL, le schéma articule également les quatre dimensions qui composent le modèle SoTL de Trigwell, Martin, Benjamin et Prosser (2000) qui, pour leur part, présentent un modèle se démarquant des 
autres par son côté multidimensionnel: 1) informer sa pratique (en orange dans le schéma) ; 2) réfléchir sur sa pratique; 3) communiquer au sujet de sa pratique; et 4) concevoir sa pratique (centrée sur l'enseignement ou sur l'apprentissage). Pour chacune de ces dimensions, les auteurs établissent quatre niveaux de positionnement des enseignants. L'échelle descriptive permet ainsi de constater, par exemple, que tel enseignant a atteint le niveau 2 en information, le niveau 3 en réflexion ainsi qu'en communication, etc. Le modèle de Trigwell et al. (2000) a l'avantage, de par son découpage en dimensions et en niveaux, de représenter une réalité moins linéaire que les autres modèles, reconnaissant ainsi qu'un enseignant ne se situe jamais de manière globale et homogène à un stade unique de développement.

\section{Le continuum des postures au savoir des enseignants et gestes d'accompagnement}

La lecture des phases de développement de la pratique de l'enseignant au sein du SoTL nous amène à les croiser avec les postures de création de savoir identifiées et caractérisées par Donnay et Charlier (2006). Ces postures ont le mérite de mettre en évidence que recherche et action se situent sur un continuum où chercheurs et praticiens peuvent entrer en relation et dégager des terrains de réflexion communs. La particularité d'une posture est qu'elle n'est pas figée comme c'est le cas d'un statut professionnel. Un enseignant peut ainsi passer d'une posture à une autre selon des moments différents et les alterner selon le contexte dans lequel il travaille. Par exemple, un enseignant, en fonction des questions pédagogiques qu'il se pose dans sa classe à différents moments de sa carrière, peut, tour à tour, être dans l'urgence de l'action, identifier les spécificités des gestes qu'il pose, adopter une posture réflexive pour construire des solutions étayées ou chercher à analyser systématiquement ces questions pour les partager ensuite avec des collègues. Donnay et Charlier (2006) placent sur un continuum non évolutif les six postures: 1) praticien, 2) praticien réfléchi, 3) praticien réflexif, 4) praticien-chercheur, 5) chercheur praticien, et 6) chercheur académique. Les postures adoptées par chacun sont dynamiques. Elles permettent de caractériser un enseignant, dans un contexte spatio-temporel spécifique. Ce dernier peut adopter des postures différentes en fonction de son groupe d'élèves, de sa trajectoire professionnelle, de son équipe ou de ses projets.

Cet apport alimente notre compréhension des caractéristiques professionnelles des enseignants aux différentes phases du SoTL. En effet, dans les postures de Donnay et Charlier, les conduites sont décrites à travers des critères pouvant refléter des sauts épistémologiques émis tant par des enseignants différents que par un même enseignant, à des moments et dans des projets différents. Le degré d'implication dans la situation, le degré de 
théorisation, le degré de communicabilité et le niveau de langage dessinent ces postures qui clarifient le processus de construction identitaire de l'enseignant dans son développement professionnel. «Le développement professionnel n'est pas une juxtaposition linéaire d'apprentissages, mais plutôt un système d'axes de développement où plusieurs facettes de la profession et de la personne interagissent entre elles et évoluent à des rythmes différents » (Donnay \& Charlier, 2006, p. 20).

\section{Référentiels de compétences pour enseignants : Quelques modèles de référence}

En vue d'une valorisation de l'enseignant-chercheur marocain comme facteur de développement et de l'acte d'enseigner comme métier à promouvoir dans la société en général et dans l'université en particulier, la définition d'un profil (ou référentiel) de compétences qui précise les axes et les diverses dimensions de la profession enseignante, s'avère indispensable et prend nombre d'utilités. Son but premier est de servir de référence, de guide, particulièrement dans un objectif d'auto-évaluation et de réflexion personnelle afin de permettre à l'enseignant ou à l'aspirant enseignant, à la lecture de l'ensemble des compétences requises à l'emploi, d'effectuer une auto-évaluation de ses compétences en réfléchissant à celles qu'il croit maîtriser (compétences identitaires), celles qu'il reconnaît comme étant en voie de maîtrise (compétences potentielles), ou à celles qu'il croit nécessaires de développer (compétences à amélioration requise) et donc de structurer son développement professionnel. Il constitue une plateforme mobilisante jouant un rôle sur le plan stratégique, opérationnel, fonctionnel, motivationnel (Foucher, 2010).

De plus, le profil de compétences vise principalement à mieux guider et professionnaliser les activités de gestion des ressources humaines telles que : le recrutement, la formation, l'évaluation, etc. il va permettre également de répondre à certains besoins de l'individu cible. Entre autres, il répond au besoin d'information, d'expression, de progression et de reconnaissance (Labruffe, 2005).

A cet effet, ce document devient le premier outil pouvant enclencher l'auto-réflexion quant aux comportements requis à l'emploi. Ainsi, il devrait être présenté à tout nouvel employé dans le processus de recrutement, d'accueil et d'intégration. D'entrée de jeu, cela permet de préciser au nouvel enseignant chercheur ce qui est attendu de lui en termes de comportements à adopter pour en arriver à Savoir agir de manière compétente. Cela permet également d'assurer au mieux la cohérence entre ce pourquoi il a été embauché, ce qui est attendu de lui, et ce sur quoi il sera évalué.

L'auto-évaluation lors de l'évaluation annuelle ou périodique dans l'organisation s'avère toute aussi importante. Elle peut permettre, à elle 
seule, de déclencher des intentions de développement chez l'individu qui s'auto-évalue avant même la transmission d'un feed-back. Ensuite, lors de la rencontre de feed-back, la confrontation des écarts entre les résultats de l'auto-évaluation et ceux produits par le supérieur ou autres personnes possédant la perspective la plus pertinente pour évaluer les comportements, permet de raffiner cette réflexion en confirmant ou infirmant certaines choses.

Nous présentons dans ce qui suit, quelques modèles de référence au Canada, aux Etats-Unis et en Bélgique en matière de référentiel de compétence visant la professionnalisation et la valorisation de la profession enseignante:

○ Le Référentiel de Compétences Professionnelles de la Profession Enseignante (Québec);

- Le Référentiel de Paquay (Communauté Française de Belgique);

$\circ \quad$ English as a new Language Standards for Teachers of Students Ages 3-18+ (Etats-Unis).

\section{Problématique \& Questions De Recherche}

La volonté de valoriser l'enseignement s'accroît de plus en plus et cette volonté est particulièrement présente dans les discours des hauts dirigeants des instances universitaires. Par conséquent, cette valorisation entraîne le maintien de standards de qualité de l'enseignement de plus en plus élevés.

Aujourd'hui, la grande majorité des processus touchant l'éducation, l'enseignement et la formation sont élaborés selon une approche compétence. Le principal avantage de cette approche est qu'elle " dépersonnalise » le processus de recrutement, de formation et d'évaluation, c'est-à-dire, qu'elle porte sur les compétences requises pour le poste ou les compétences qu'un individu devrait posséder pour s'enquérir de ses tâches et remplir sa fonction plutôt que de porter sur la mesure quantitative ou qualitative de la capacité de l'individu à atteindre les objectifs qui lui ont été fixés. Ainsi, l'évaluation effectuée dans une perspective compétencielle devrait davantage viser le développement des compétences requises plutôt que de remettre en question, par des mesures perceptuelles qualitatives ou quantitatives, la capacité de l'individu à atteindre lesdits objectifs pour l'accomplissement de sa tâche. Or, la perception devient davantage développementale et moins administrative, ce qui contribuerait à rendre le feedback plus accepté et utilisé par l'individu cible (Brassard, 2009; Foucher, 2010). Par conséquent, l'approche axée sur les compétences permet également une meilleure responsabilisation des individus puisqu'elle vise principalement, voire uniquement, le développement individuel. Elle est donc davantage perçue comme non menaçante (Brassard, 2015). 
Dans un contexte marocain, la grande majorité des institutions universitaires et plus précisément les grandes écoles de commerce et d'ingénieur (objet de notre étude), l'évaluation des enseignements s'effectue de manière non homogène, plus ou moins en référence avec des normes, standards, critères et objectifs clairement établis. De plus, lorsqu'il y en a, dans lesdites institutions, les indicateurs à partir desquels un enseignant chercheur devrait être recruté, formé et évalué ne sont pas présentés aux principaux intéressés ou encore, ne s'inscrivent pas à l'intérieur d'une démarche cohérente visant le développement professionnel. Au terme de quelques entretiens prliminaires effectuées, il en est ressorti, que de nombreux enseignants-chercheurs marocains mentionnent ne posséder aucun outil leur permettant d'être guidés dans le développement de leurs compétences. Il semblerait que la seule forme d'évaluation de ces enseignants soit effectuée principalement à des fins administratives. Plusieurs précisent également ne pas recevoir de feedback, d'autres le qualifient d'inadéquat ou encore jugent que la rétroaction qu'ils reçoivent n'est pas livrée en temps opportun ou ne s'avère pas vraiment utile pour l'amélioration de la qualité des enseignements.

$\mathrm{Au} v \mathrm{vu}$ du contexte décrit précédemment, notre problématique peut se résumer globalement dans la question suivante:

\section{Dans quelle mesure la professionnalisation de la GRH influence-t-elle la valorisation du métier de l'enseignant-chercheur ? Quel rôle de l'approche compétence?}

Trois principales questions découlent de cette problématique:

1) Quels sont les facteurs déterminants qui influencent la valorisation du métier de l'enseignant-chercheur au Maroc ?

2) Quel impact des pratiques $\mathrm{RH}$ sur le processus de professionnalisation du personnel enseignant ? et comment interviennentelles pour le juger comme étant un enseignant de niveau universitaire de valeur?

3) A la lumière de ces éléments, comment définir l'enseignantchercheur marocain en matière d'identité professionnelle et de compétences à développer? Quels indicateurs comportementaux permettront de juger le degré d'atteinte de chacune de ces compétences?

\section{Méthodologie de la recherche \\ Positionnement épistémologique}

Comme le souligne Bourdieu (1987), la réflexion épistémologique, en ce qu'elle invite à expliciter les présupposés et justifier les choix effectués à ces différentes étapes, est en outre un puissant outil d'innovation pour la recherche en permettant de dépasser la simple recherche de cohérence entre l'analyse et les objets de cette analyse. Cette posture réflexive offre au 
chercheur les outils d'une pratique scientifique consciente d'elle-même et contrôlée, "pour lutter contre les contraintes de l'espace théorique du moment et pour dépasser les prétendues incompatibilités, les prétendues oppositions, les prétendues voies inconciliables »

L'épistémologie s'intéresse principalement aux trois questions suivantes (Perret et Séville, 2007) : quelle est la nature de la connaissance produite ? Comment la connaissance est-elle engendrée, quels chemins a-t-elle emprunté ? Quels sont les critères de validité de cette connaissance?

Gavard-Perret et al. (2012) soulignent trois types de positionnement que le chercheur peut suivre dans son travail de recherche. Il s'agit d'un positionnement unique, multiple ou aménagé. Le positionnement unique consiste à choisir un des paradigmes épistémologiques et de l'appliquer d'une manière stricte et rigoureuse. Le deuxième positionnement (multiple) considère qu'un dialogue entre plusieurs paradigmes est possible pour améliorer la compréhension des phénomènes sociaux.

Koenig (1993) postule en effet que c'est une opportunité pour le chercheur de s'enrichir de la diversité et de la pluralité des paradigmes pour rendre compte des réalités complexes.

Enfin, le troisième type de positionnement (paradigme aménagé) consiste à intégrer différentes postures épistémologiques contrastées à travers lesquelles les efforts d'intégration portent sur la recherche d'un standard commun. Thietart (2014) souligne que de nombreuses recherches empruntent effectivement des éléments relatifs à différents paradigmes.

Dans le cadre de cette thèse, nous avons opté pour ce troisième type de positionnement et, plus précisément, pour un positionnement positiviste aménagé. Miles et Huberman, souvent cités en appui de cette position, considèrent « que les phénomènes sociaux existent non seulement dans les esprits mais aussi dans le monde réel et qu'on peut découvrir entre eux quelques relations légitimes et raisonnablement stables ». En effet, nous allons dans un premier temps procéder à une analyse de la littérature relative aux concepts thèse (valorisation du métier de l'enseignant-chercheur, professionnalisation de la GRH et approche compétence) et les modèles de référence mobilisés afin de proposer à l'issu de cette analyse un cadre conceptuel permettant de répondre à notre problématique. Dès lors, cette recherche s'inscrit dans une approche hypothético-déductive dont le choix est justifié par les objectifs que nous avons fixés : explorer, décrire et vérifier. Toutefois, avant de passer à l'étude quantitative, nous aurons recours à une étude qualitative de type exploratoire afin d'affiner et vérifier la pertinence de notre modèle de recherche. Ce mode de réflexion consiste à découvrir des régularités à partir de l'observation de l'objet puis à formuler des hypothèses dans une perspective inductive, les hypothèses qui vont être 
retenues étant en partie issues du terrain et influencées par lui (Thietart, 2014).

En combinant une réflexion hypothético-déductive et une réflexion inductive dans notre recherche, nous nous positionnons dans le courant du positivisme aménagé. Les facteurs liés à la professionnalisation de la GRH à travers une approche compétence existent, mais les attitudes envers ces facteurs seront différentes en fonction de profil et de personnalité de l'individu cible qu'est l'enseignant-chercheur. Ainsi, nous considérons que la réalité existe, mais qu'elle peut être « aménagée » en fonction du contexte, des situations, des personnes et des interprétations (Gavard-Perret et al., 2012).

\section{Recherche qualitative exploratoire Objectifs de l'étude qualitative}

L'étude qualitative possède une double fonction : confirmatoire et exploratoire.

Elle est confirmatoire puisqu'elle permettra de vérifier l'exactitude des éléments issus de la revue de la littérature. En effet, cette dimension confirmatoire a pour objectif de s'assurer que les principaux concepts mentionnés par la littérature se retrouvent dans le discours des interviewés. Elle est également exploratoire puisqu'elle permettra d'enrichir les résultats de la revue de la littérature.

Il est vrai que la vocation principale d'une étude qualitative est la production de nouvelles informations et de mettre en évidence et de comprendre les motivations et comportements des individus. Ces deux dimensions, confirmatoire et exploratoire de l'étude qualitative seront également utilisées afin de générer les items de nos échelles de mesure.

La combinaison des approches qualitatives et quantitatives et le recours à la triangulation des méthodes de collecte et d'analyse des données sont particulièrement recommandées quand on s'intéresse à un champ de recherche relativement nouveau (Beail et Williams, 2014). La valorisation du métier de l'enseignant-chercheur étant un champ très peu investigué, et les méthodes qualitatives sont mieux adaptées pour appréhender la complexité du métier et mesurer l'impact de la professionnalisation des enseignantschercheurs sur sa valorisation et l'amélioration de son attractivité.

En résumé, cette phase qualitative est utilisée d'une part dans une optique séquentielle (Thietart, 2014) où elle devance la phase quantitative offrant une meilleure appréhension du sujet de recherche et la proposition $d$ 'hypothèses de recherches et d'autre part, dans une optique de triangulation des données (Thiétart, 2014) en permettant de confirmer ou non certaines hypothèses sous-jacentes aux éléments avancés dans la littérature. 


\section{Méthode de collecte de données: L'entretien semi-directif}

Dans le cadre de notre future étude exploratoire, des entretiens individuels semi-directifs seront conduits. Contrairement aux réponses obtenues par questionnaire, l'entretien semi-directif se caractérise par l'absence de préformation permettant ainsi d'obtenir des informations symptomatiques, profondes et très riches.

L'approche par entretien semi-directif s'avère particulièrement adéquate pour étudier les moyens de valorisation du métier de l'enseignantchercheur au Maroc. Elle est aussi utile pour comprendre et interpréter le sens que donne les enseignants à leurs attentes, aspirations et carrières.

Cette méthode permet à l'individu de raconter l'historique et les différentes phases de sa carrière. En le faisant, il décrit les différentes expériences qu'il a vécu, non nécessairement chronologique. Il peut aussi révéler des incohérences et contradictions liées à son expérience de travail. Le récit de vie implique une réorganisation d'événements du passé, auxquels l'individu interrogé attribue du sens (Burrick, 2010).

L'entretien semi-directif nécessite l'utilisation d'un guide d'entretien listant les thèmes à aborder durant l'entretien. La technique employée consiste à poser initialement une question ouverte à l'interviewé, à le laisser parler, tout en le relançant sans pour autant diriger ses propos. Certains thèmes peuvent être introduits par le répondant au cours de l'entretien soit parcequ'ils n'ont pas été abordés spontanément par l'enquêté soit pour les approfondir.

Nous allons privilégier d'attacher une certaine attention à chaque entretien pris en particulier sans pour autant négliger la mise en relation de tous les entretiens entre eux dans le but de répondre aux objectifs de la recherche.

\section{Démarche quantitative de l'étude empirique Justification de la démarche de recherche}

Sur la base de l'analyse de la littérature et du cadrage conceptuel de la présente recherche, nous allons proposer des hypothèses de recherche. Cette littérature sur les concepts de cette thèse permet d'avoir une approche hypothético-déductive. Comme le souligne Roussel (2005): " lorsque les travaux théoriques procurent assez de connaissances sur le phénomène étudié et posent des bases théoriques intellectuellement cohérentes et empiriquement pertinentes (en fonction des entretiens et études de cas réalisés précédemment, ou des travaux publiés dans les revues scientifiques), l'approche déductive est souhaitable ». En effet, la pléthore de travaux sur la professionnalisation de la GRH, l'approche compétence, la valorisation du métier de l'enseignant-chercheuret et sur la nature des liens entre ces variables permet de construire un modèle conceptuel de base qui sera testé 
dans ladite étude empirique quantitative.

\section{L’échantillon de l'étude}

La population étudiée dans le cadre de cette recherche est constituée des enseignants-chercheurs marocains. Cependant, cette population est en réalité très vaste et ne peut être interrogée dans sa totalité (dans l'absence d'une base de sondage officielle et exhaustive). Notre choix sera donc orienté vers un échantillon des enseignants-chercheurs des grandes écoles d'ingénieur et de commerce de l'enseignement supérieur marocain relevant ou non des universités.

\section{Modèle conceptuel de recherche}

Pour définir notre modèle conceptual de recherche, une recension des différents écrits scientifiques, des documents, des études de reference et des sites web de plusieurs institutions universitaires de renom à l'échelle international a d'abord été réalisée pour nous permettre d'établir un modèle initial, mais nous trouvons plus productif de procéder à une étude exploratoire qui va nous permettre d'adapter notre modèle de recherche initial, puisqu'elle va nous amener à avoir une vision globale sur le métier de l'enseignant-chercheur pour bien le définir en matière d'identité professionnelle et ses attentes et aspirations, en tenant compte des dimensions pédagogiques, institutionnels et socioprofessionnelles afin de le juger par la suite comme étant un enseignant de niveau universitaire de valeur. Cette première phase de travail a abouti au modèle conceptuel cidessous: 
Figure 5. Cadre conceptuel de la recherche

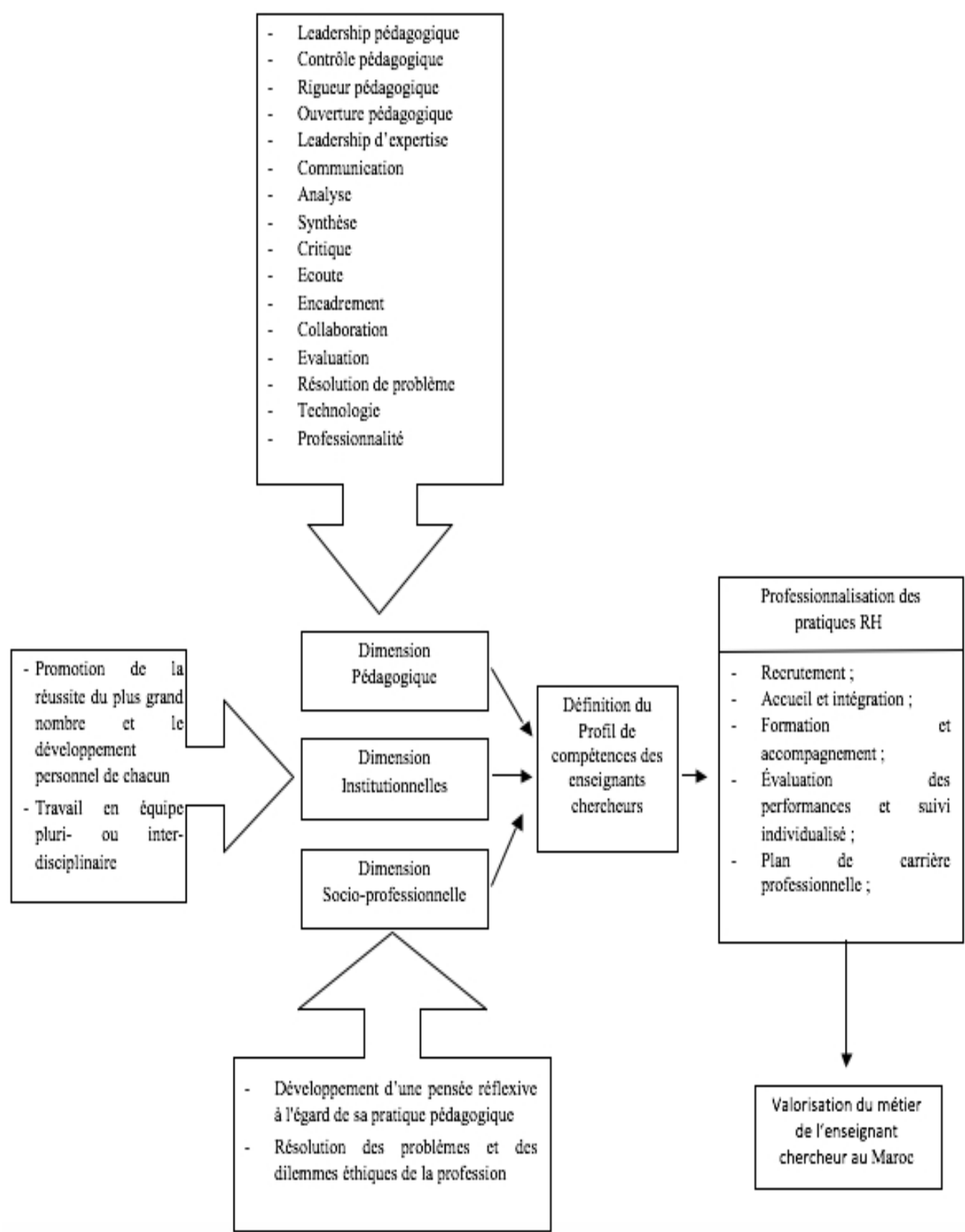

\section{Démarche de recherche}

Pour répondre à la problématique fixée pour cette recherche, nous devons établir une démarche de recherche qui va consister en cinq étapes résumées par la figure suivante: 
Figure 6. Démarche scientifique globale de notre recherche

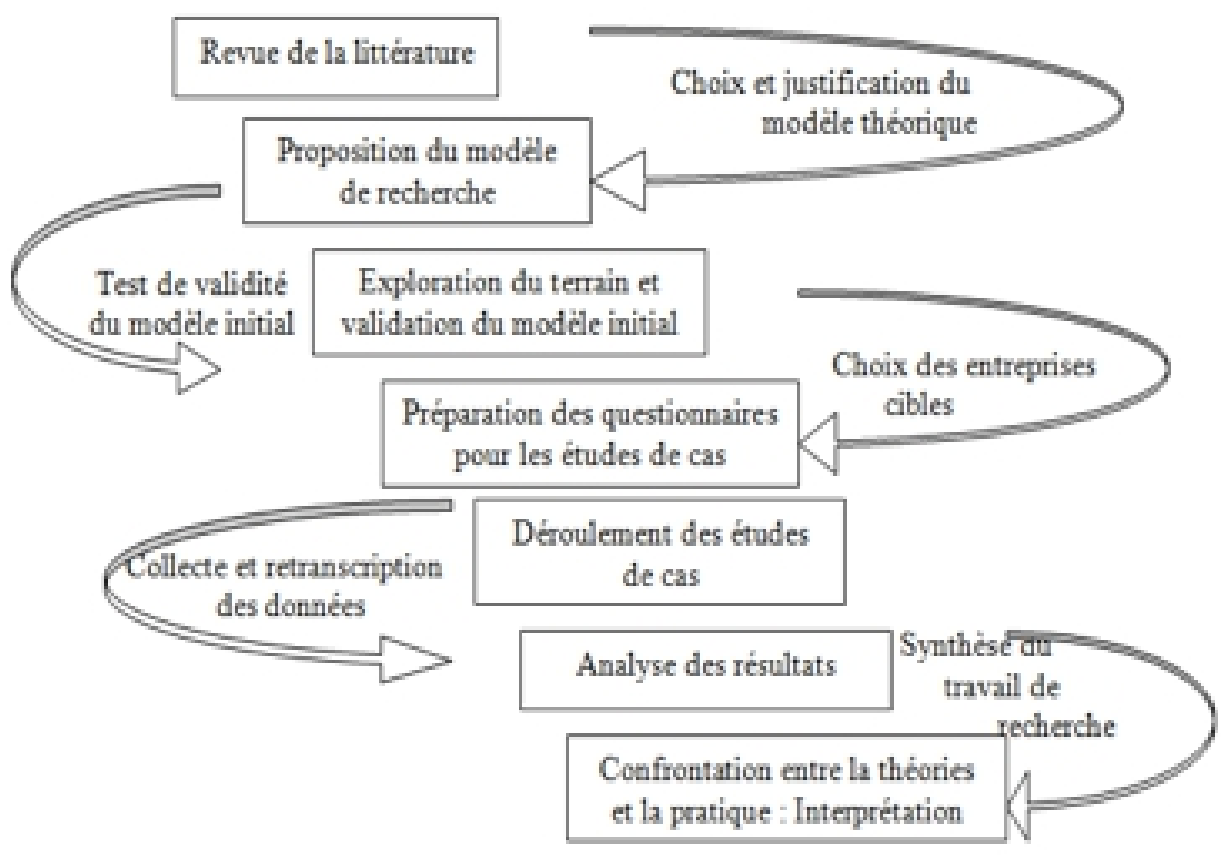

Notre problématique de recherche étant établie, nous procéderons dans un premier temps à une analyse de la littérature afin de ressortir notre cadre conceptuel. Une lecture transversale de la littérature nous a permis de repérer un ensemble de modèles de référence sur la valorisation du métier de l'enseignant-chercheur. Cependant en ce qui concerne les facteurs liés à la professionnalisation de la GRH à travers une approche compétence, la littérature n'offre pas d'une manière précise, les facteurs susceptibles d'influencer la valorisation du métier de l'enseignant-chercheur. Les facteurs liés à la GRH sont nombreux, il nous est impossible de les retenir tous. Dès lors, nous allons opté pour une étude qualitative exploratoire afin de mieux cadrer notre cadre conceptuel. En combinant les deux approches qualitative et quantitative, le chercheur peut garantir au mieux la validité des résultats (Thietart, 2014). La complémentarité de ces deux approches qualitatives et quantitatives est devenue indispensable dans les travaux de recherche. L'étude qualitative constitue souvent un préalable indispensable pour tout chercheur qui sera amené par la suite à faire une étude quantitative. Elle permet de recadrer et affiner la question de recherche, elle permet au chercheur de se familiariser avec le sujet en se mettant dans la réalité des choses, elle permet aussi de clarifier les concepts théoriques de la recherche. L'étude qualitative présentera également un intérêt pour préciser les énoncés des instruments de mesure. 
La combinaison entre l'analyse de la littérature et l'étude qualitative va nous permettre de construire et de valider notre modèle de recherche.

Le modèle enrichi et les hypothèses de recherche seront alors testés dans une étape quantitative. Cette phase quantitative est indispensable pour généraliser les caractéristiques observées sur notre population de l'étude qualitative. L'analyse des résultats nous permettra de formuler un certain nombre de conclusions relatives à notre problématique de recherche.

Nous croyons que cette démarche a ses avantages puisqu'elle allie

la théorie à la pratique. De plus, le chercheur et les enseignants collaborent dans le processus de recherche. En d'autres termes, cette approche prend en considération les besoins et les attentes des enseignants chercheurs eux-mêmes ainsi que la spécificité du contexte, ce qui peut garantir son efficacité, sa validité ainsi que sa représentativité.

Nous résumons ci dessous l'articulation de notre démarche en distinguant ce qui est à décrire et à expliquer, ce qui se traduit en variables mesurables et les modes d'investigation choisis pour l'étude emprique.

Figure 7. L'articulation du sujet, de l'objet d'étude et des modes opératoires

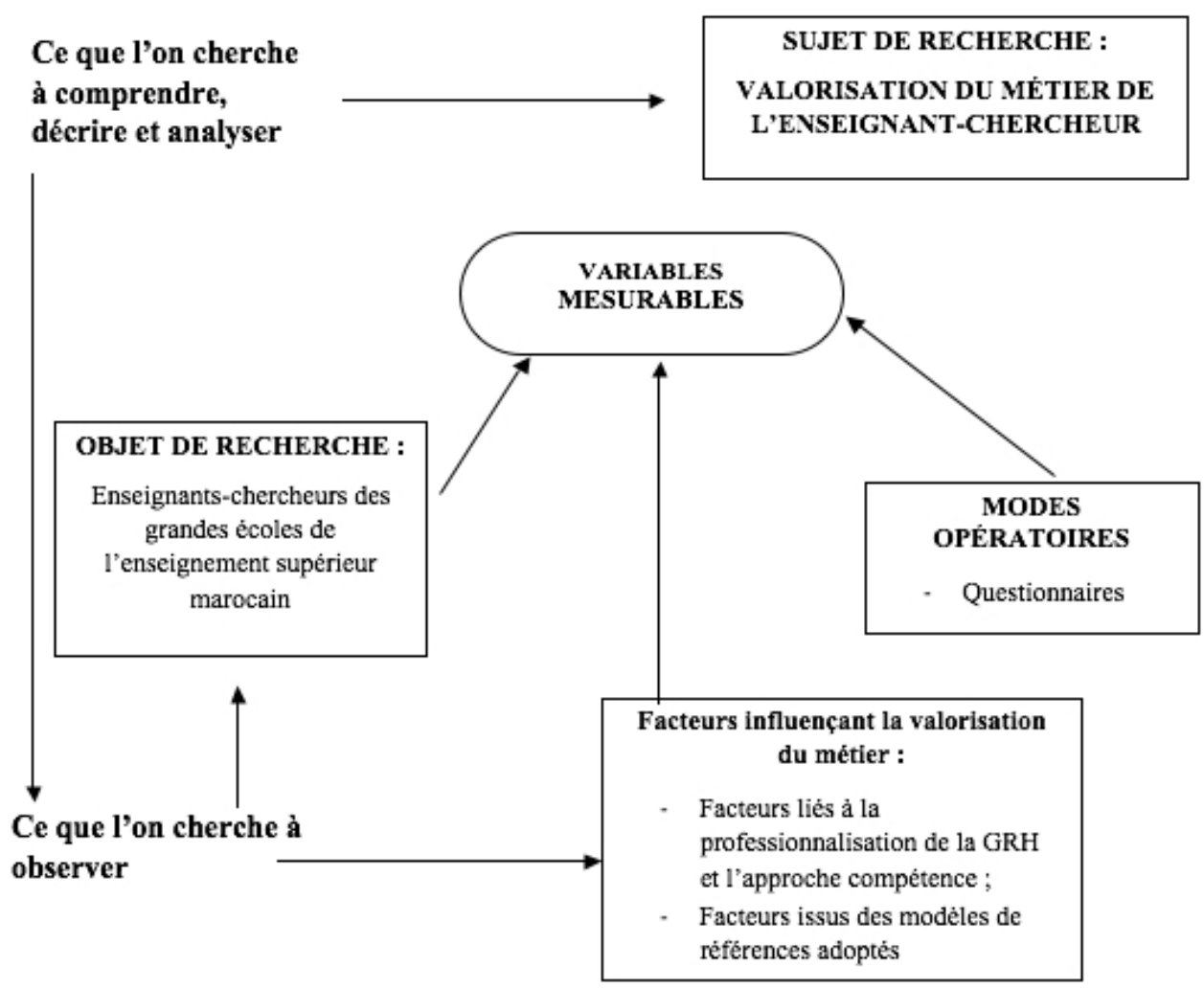




\section{Conclusion \& projection}

Les référentiels de compétences et le développment professionnelle des enseignants sont devenus des sujets qui suscitent beaucoup d'intérêt de la part des chercheurs en éducation (Paquay, 1994; Perrenoud, 1994; Perrenoud, 2001; Berchoud, 2007). D'ailleurs, nous repérons beaucoup de référentiels développés dans plusieurs pays du monde pour guider le développement professionnel des enseignants comme c'est le cas en France, en Belgique, au Québec en particulier.

A notre connaissance, cette recherche est la première tentative visant la compréhension de la question de la GRH, comme élément de professionnalisation et de valorisation de l'attractivité du métier de l'enseignant chercheur au Maroc. En d'autres termes, une approche compétence du management des ressources humaines personnalisée aux enseignants des grandes écoles va ouvrir une véritable perspective opérationnelle aux décideurs, mais aussi la méthodologie que nous allons developer, constitue une des principales contributions de cette rechercheaction.

Les prochaines étapes de notre recherche consisteront à entamer la phase de validation empirique de notre modèle de recherche auprès des enseignants-chercheurs des grandes écoles d'ingénieur et de commerce au Maroc et de produire les hypothèses de recherche y afférents. Cela pourrait permettre de dégager de nouvelles variables et de recueillir d'eventuels commentaires pour procéder ainsi à la modification de quelques variables non pertinentes.

\section{References:}

1. Albero Brigitte, R. «Professionnaliser les enseignants-chercheurs à l'université : les effets pervers d'une bonne idée» http://dms.revues.org/1124

2. Bailly Basile, Demougeot-Lebel Joëlle \& Lison Christelle « La formation d'enseignants universitaires nouvellement recrutés : quelles retombées ? » https://ripes.revues.org/990\#tocfrom1n1

3. Beacco, J. C. (2007). L'approche par compétences dans l'enseignement. Enseigner à partir du Cadre européen commun de référence pour les langues. Paris : Didier.

4. Beckers, J. (2007). Compétences et identité professionnelles : l'enseignement et autres métiers de l'interaction humaine. Bruxelles : De Boeck.

5. Berchoud, M. (2007). Vers un référentiel de compétences pour les enseignants en FLS- FLE : Langue, culture, relations...en France - et au Québec? AQEFLS, 26(2), 66- 81. 
6. Blais, J. G., Laurier, M. D., \& Rousseau, C. (2006). La construction de compétences à partir d'indicateurs de pertinence. In G. Figari, P. Rodrigue, \& M. Palmira Alve (dir.) Évaluation des compétences et apprentissages expérientiels. Savoirs, modèles et méthodes (pp. 349361). Lisbonne : EDUCA

7. Brassard, N. (2016). «Développement des compétences de l'enseignant de niveau universitaire : Outil d'auto-évaluation et de diagnostic professionnel ». Montréal, ENAP.

8. Brassard, N. (2012). «Profil de compétences de l'enseignant de niveau universitaire ». Portail du soutien à la pédagogie universitaire du Réseau de l'Université du Québec http://pedagogie.uquebec.ca/portail/repertoire/profil-de-competencesde-lenseignant-de-niveau-universitaire

9. Bru, M., \& Talbot, L. (2007). Des compétences pour enseigner : entre objets sociaux et objets de recherche. Rennes : Presses universitaires de Rennes.

10. Donnay Jean \& Romainville Marc (éds.) (1996). Enseigner à l'Université, un métier qui s'apprend? Bruxelles : De Boeck, 155 pges.

11. Ebtehal Abdel Moneim Mahrousse Hussein. Thèse : «Élaboration d'un référentiel de compétences pour les futurs enseignants de FLE », Université de Montréal, Faculté des études supérieures et postdoctorales.

12. Endrizzi Laure. «Savoir enseigner dans le supérieur: un enjeu d'excellence pédagogique ». Dossier de veille de l'IFÉ http://ife.enslyon.fr/vst/DA/detailsDossier.php?dossier=64\&lang=fr

13. Figari, G. (2006b). Les référentiels entre théorie et méthodologie. In G. Figari, \& L. M. Lopez (dir.), Recherche sur l'évaluation en éducation. Problématiques, méthodologies et épistémologie (pp. 101108). Paris : L'Harmattan.

14. Hensler, H., \& Desjardins, J. (2006). Pourquoi l'approche par compétences oblige-t-elle à reconsidérer le rôle de la réflexion dans la formation des enseignants? In J. Desjardins, H. Hautes écoles pédagogiques (s. d.). Formation des enseignants. Référentiel de compétences professionnelles. Suisse : Direction de la formation. $\begin{array}{lllll}\text { Consulté } & \text { le } & 20 & \text { juillet } & 2010\end{array}$ http://www.hepl.ch/files/live/sites/systemsite/files/interfilieres/refer entiel-competences-hep-vaud.pdf

15. Le Boterf, G. (2002). Développer la compétence des professionnels. Paris : Édition d'organisation.

16. Ministère de la Communauté Française (2001). Devenir enseignant. Le métier change, la formation aussi [fascicule]. Bruxelles : CFWB. 
Consulté le $\quad 25 \quad$ juillet 2008

http://www.enseignement.be/download.php?do_id=1355

17. Ministère de l'Éducation, du Loisir et du Sport (2006). Échelles des niveaux de compétence : enseignement secondaire : premier cycle. Québec : Le Ministère.

18. Nagels Marc \& Le Goff Morgane. «DES REFERENTIELS DE COMPETENCES INNOVANTS : Quelle appropriation par les enseignants ? » Université Nanterre - Paris École des hautes études en santé publique (EHESP); https://halshs.archives-ouvertes.fr/hal00287594/document

19. National Standards in Foreign Language Education Project (1999). Standards for foreign language learning in the 21st century. USA : National Standards in Foreign Language Education Project.

20. Paquay, L. (1994). Vers un référentiel des compétences professionnelles de l'enseignant? Recherche et formation, 16, 7-38.

21. Paquay, L. (1998). Évaluation et formation des enseignants. Louvainla-Neuve : Academia-Bruylant.

22. Paquay, L. (2002). L'évaluation des compétences chez l'apprenant : pratiques, méthodes et fondements. Louvain-la-Neuve : UCL presses universitaires de Louvain.

23. Paquay, L. (2004). L'évaluation des enseignants et de leur enseignement : Pratiques diverses, questions multiples. In L. Paquay (dir.), L'évaluation des enseignants : tensions et enjeux (pp. 13-57). Paris : L'Harmattan.

24. Paquay, L. (2006). L'évaluation des enseignants, en tensions et en perspectives. In G. Figari, \& L. M. Lopez (dir.), Recherche sur l'évaluation en éducation. Problématiques, méthodologies et épistémologie (pp. 51-58).Paris: L'Harmattan.

25. Paquay, L., Altet, M., \& Perrenoud, P. (1998). Former des enseignants professionnels : trois ensembles de questions. In $\mathrm{L}$. Paquay, M. Altet, \& P. Perrenoud (dir.), Former des enseignants professionnels : Quelles stratégies? Quelles compétences? (pp.13-26). Bruxelles : De Boeck.

26. Parmentier, C. (2005). Les 10 compétences de l'enseignant universitaire. Cahiers de L'Institut de pédagogie universitaire et des multimédias. Université catholique de Louvain.

27. Peraya Daniel. «Professionnalisation et développement professionnel des enseignants universitaires: une question d'actualité » https://dms.revues.org/1094\#tocto2n1

28. Périsset Bagnoud, D. (2007). Les référentiels de compétences, profils attendus de la professionnalité enseignante. Acteurs et points de vue en Suisse romande. In M. Bru, \& L. Talbot (dir.), Des compétences 
pour enseigner : entre objets sociaux et objets de recherche (pp.87104). Rennes : Presses universitaires de Rennes.

29. Postiaux. N, Bouillard. P \& Romainville. «Référentiels de compétences à l'université : Usages, rôles et limites »; http://rechercheformation.revues.org/185

30. Postiaux, N. \& Romainville, M. « La compétence asservit-elle l'Université au monde professionnel, la faisant ainsi renoncer à son idéal pédagogique ?» Université libre de Bruxelles ; http://www.univ-corse.fr/docs/ndoc1928.pdf

31. Savard Claude, Lessard Johanne, Paquet Manon, Bilodeau RenéeClaude; Faucher Alain, Talbot Serge, «Enseigner à l'Université Laval : un référentiel de compétences en pédagogie universitaire », Université Laval (Canada); https://prezi.com/rovvoywov7if/enseigner-a-luniversite-laval-unreferentiel-de-competences-en-pedagogie-universitaire/ ou https://oraprdnt.uqtr.uquebec.ca/pls/public/docs/GSC2220/F9430134 93_PROGRAMME_COMPLET_ET_ACTES_Communications_indi viduelles_session_7_15_Version_finale.pdf. 